\title{
Update on denosumab in the management of postmenopausal osteoporosis: patient preference and adherence
}

This article was published in the following Dove Press journal:

International Journal of Women's Health

13 October 2015

Number of times this article has been viewed

\author{
Elisa Cairoli ${ }^{1,2}$ \\ Cristina Eller-Vainicher ${ }^{1}$ \\ lacopo Chiodini ${ }^{1,2}$ \\ 'Unit of Endocrinology and Metabolic \\ Diseases, Fondazione IRCCS Ca' \\ Granda - Ospedale Maggiore \\ Policlinico, Padiglione Granelli, Milan, \\ Italy; ${ }^{2}$ Department of Clinical Sciences \\ and Community Health, University of \\ Milan, Milan, Italy
}

\begin{abstract}
Patient adherence to many osteoporosis treatments, primarily bisphosphonates, is generally poor, thus leading to a significant reduction in antifracture efficacy. Patient perceptions about the necessity of the prescribed medication to treat osteoporosis and the concerns about the potential adverse effects are important and potentially modifiable determinants of adherence, in addition to other factors, such as difficult dosing regimens and high dosing frequency. Denosumab (Dmab) is a fully human monoclonal antibody against the receptor activator of nuclear factor- $\mathrm{\kappa B}$ ligand (RANKL), which, through the prevention of the RANKL/RANK interaction, inhibits osteoclast-mediated bone resorption and significantly reduces the risk of vertebral, nonvertebral, and hip fractures. It is administered subcutaneously every 6 months for the treatment of postmenopausal osteoporosis. Preference and adherence to Dmab treatment were assessed in various clinical trials. Although with some limitations, available data suggest that Dmab is preferred to bisphosphonates, produces greater satisfaction than bisphosphonates, and would be preferentially chosen for long-term treatment. Moreover, patient perceptions about the necessity of Dmab treatment clearly outweigh the concerns about the injections, and positive beliefs about treatment positively influence medication-taking behavior. According to these data, Dmab may represent a reasonable alternative to bisphosphonates, particularly for osteoporotic women in whom a suboptimal or even poor adherence to oral treatments is expected.
\end{abstract}

Keywords: denosumab, RANKL, osteoporosis treatment, preference, satisfaction, adherence

\section{Introduction}

Denosumab (Dmab) is a fully human monoclonal antibody against the receptor activator of nuclear factor- $\mathrm{KB}$ ligand (RANKL), a molecule that is crucial for the formation, function, and survival of osteoclasts. ${ }^{1}$ Dmab binds RANKL with high affinity and specificity, and inhibits the RANKL/RANK interaction, thus reversibly reducing osteoclast-mediated bone resorption. In phase 1 and phase 2 studies, Dmab was demonstrated to decrease bone turnover and to increase bone mineral density (BMD), ${ }^{2-5}$ and in the FREEDOM trial (Fracture REduction Evaluation of Denosumab in Osteoporosis every six Months, NCT00089791), a randomized placebo-controlled phase 3 study, the subcutaneous administration of Dmab $60 \mathrm{mg}$ every 6 months for 36 months significantly reduced the risk of vertebral and nonvertebral fractures, and reduced the risk of hip fracture in postmenopausal women with osteoporosis. ${ }^{6}$ Moreover, in the open-label extension of this study, Dmab therapy beyond the third year of treatment was associated with a further reduction in nonvertebral fracture rate, and was associated with a continued low vertebral fracture rate that persisted through 8 years of continuous administration, with an overall safety profile that remained consistent
Correspondence: Elisa Cairoli Unit of Endocrinology and Metabolic Diseases, Fondazione IRCCS Ca' Granda - Ospedale Maggiore Policlinico, Padiglione Granelli, Via F Sforza 35, 20122 Milan, Italy

Tel +390255033355

Fax+390250320605

Email elisa.cairoli@live.it 
over time. ${ }^{7}$ On the basis of available evidence, in 2010 , Dmab was approved for the treatment of postmenopausal osteoporosis, thus becoming a further therapeutic option for the reduction of fracture risk in addition to the other available antiresorptive therapies (ie, bisphosphonates and selective estrogen receptor modulators) and the anabolic teriparatide. ${ }^{8}$ The available studies comparing the effect on BMD and bone turnover of Dmab and bisphosphonates, which are the most frequently used agents for the management of osteoporosis, showed significantly greater gains in BMD at all measured skeletal sites ${ }^{9-13}$ and greater reduction in bone turnover ${ }^{9-12}$ with Dmab compared to bisphosphonates with a similar safety profile. However, both bisphosphonates and Dmab, in association with calcium and vitamin $\mathrm{D}$, appear to be about equally effective in clinical trials in reducing the risk of fragility fractures, ${ }^{14}$ which represent a considerable problem of public health, considering the increasing fracture-related morbidity, mortality, and medical costs in many regions of the world. ${ }^{15}$

It must be considered, however, that any therapy, even if proved to be effective in clinical trials, requires adherence to achieve successful treatment outcomes. Adherence is a term that usually encompasses two different concepts: persistence and compliance. Persistence is the duration of time from initiation to discontinuation of therapy, while compliance is the degree to which a patient takes the medication as prescribed. ${ }^{16}$ Accordingly, nonpersistence and noncompliance are usually defined as a gap in therapy greater than 90 days and a medication taken less than $80 \%$ of possible treatment days, respectively. ${ }^{16}$ Adherence to osteoporosis treatments is particularly challenging for health care professionals treating osteoporosis. Indeed, persistence and compliance with osteoporosis therapies are generally poor, thus leading to a significant reduction in their antifracture efficacy, ${ }^{17}$ which in turn leads to increased human and economic costs. ${ }^{18}$ In order to understand the extent of the problem, it is worth explaining that previous studies showed that one-third to one-half of treated patients are not adherent to oral bisphosphonate treatment, ${ }^{19}$ and that the majority of patients discontinue oral bisphosphonate treatment within 1 year, ${ }^{17,19}$ with a mean persistence of only 184 days. ${ }^{17}$ In comparison with oral dosing regimens, persistence seems to be greater with an intravenous bisphosphonate administered less frequently, like the annual infusion of zoledronic acid, but it is anyway suboptimal. Indeed, a variable proportion of patients from one-third to two-thirds across studies did not receive a second administration of the drug, often because of adverse effects (postinfusion syndrome). ${ }^{20-22}$ These findings are due to the fact that treatment adherence among patients with chronic diseases like osteoporosis depends on various factors, among which difficult dosing regimens, high dosing frequency, and the occurrence of side effects play a significant role in reducing compliance and persistence. Moreover, patient perception about the necessity of the prescribed medication to treat osteoporosis and their concerns about potential adverse effects are important and potentially modifiable determinants of adherence, especially if clarified and addressed at the beginning of the treatment. Finally, understanding patient preference may be a strategy to improve adherence to osteoporosis therapy, since a lower treatment satisfaction is associated with an increased risk of discontinuing or switching the ongoing osteoporosis medication, as compared with a higher treatment satisfaction. ${ }^{23}$

In this review, we will focus on the results of studies that investigated patient preference and adherence to Dmab for the treatment of postmenopausal osteoporosis in comparison with alternative osteoporosis therapies, especially bisphosphonates, in order to establish who can take more advantage of Dmab therapy, to understand the possible factors that influence medication-taking behavior, and to discover potential strategies for improving adherence.

\section{Patient preference for Dmab}

Patient preference to and satisfaction with a specific drug are important determinants of adherence to therapies for chronic diseases, including osteoporosis. ${ }^{23,24}$ Preference is a relative index of desirability, and it can be measured as a choice between alternatives or scaled as a degree of desirability, ${ }^{25}$ while treatment satisfaction measures the degree to which patient expectations with different features of the ongoing treatment (eg, perceived efficacy, presence and severity of side effects, convenience, and bother with treatment) are met. ${ }^{25}$

Available studies typically compared patient preference to and satisfaction with Dmab versus bisphosphonates, especially alendronate, which is usually the first-line medication for the treatment of postmenopausal osteoporosis. ${ }^{26-28}$ Since existing questionnaires assessing preference to and satisfaction with osteoporosis treatments were considered inadequate for the comparison between a weekly oral tablet and a 6-monthly subcutaneous injection, a new tool, the Preference and Satisfaction Questionnaire (PSQ), was developed to compare Dmab and alendronate. ${ }^{25}$ The PSQ consists of 34 items that explore preference (the treatment choice made by a patient), satisfaction (the degree to which the features of a specific drug actually meet the patient expectations), and 
finally, bother (the degree to which the patient is disturbed by certain features of the treatment). ${ }^{25}$

In the Determining Efficacy: Comparison of Initiating DEnosumab versus alendronate (DECIDE) trial and the Study of Transitioning from AleNdronate to Denosumab (STAND), two international, double-blind, double-dummy, randomized, phase 3 head-to-head trials comparing Dmab with alendronate, ${ }^{9,10}$ PSQ was completed after 12 months of treatment or upon study discontinuation. ${ }^{26}$ Among the subjects who expressed a preference, significantly more patients, who were blinded to their treatment assignment, preferred the injection over the tablet, and were more satisfied overall and with the dosing frequency of a 6-monthly injection over a weekly tablet after 12 months of treatment. Moreover, more patients indicated that they would choose the 6-monthly injection, which was better fitted to their lifestyles, for long-term use or continuation of treatment. Finally, among patients who expressed bother with treatments, more patients found that the weekly tablet was more bothersome than the 6-monthly injection. ${ }^{26}$

A subsequent multicenter, randomized, open-label, 2-year crossover trial, the Denosumab Adherence Preference Satisfaction (DAPS) study, ${ }^{28}$ enrolled drug-naïve postmenopausal women with low BMD, who were randomized in one of two treatment sequences: Dmab subcutaneously every 6 months for 1 year followed by alendronate orally once weekly for 1 year, or vice versa. At each follow-up visit, subjects completed questions about preference, satisfaction, and bother, which were taken from the PSQ. At baseline and at 6 months, subjects reported lower mean scores concerning preference for alendronate than for Dmab, at 12 months significantly more subjects treated with Dmab than with alendronate reported to be either satisfied or quite satisfied with the dosing frequency, route of administration, convenience, and expressed overall satisfaction with the ongoing Dmab treatment. ${ }^{27}$ The final results from both years of the DAPS study further confirmed the data obtained before the crossover: at the end of the study, $92.4 \%$ of subjects preferred subcutaneous Dmab injections over alendronate tablets, and $91.2 \%$ of subjects said that they would choose Dmab injections for long-term treatment. In addition, at 24 months, regardless of the treatment sequence, a greater proportion of subjects reported that they were quite/very satisfied with the attributes of Dmab compared with those of alendronate. ${ }^{28}$

A recent study evaluated the change in treatment satisfaction in women with postmenopausal osteoporosis who were suboptimally adherent with prior daily or weekly bisphosphonate therapy and who were shifted to subcutaneous 6-monthly Dmab or monthly oral bisphosphonate (ibandronate or risedronate). ${ }^{29}$ In such study, a post hoc analysis of the results of two international, multicenter, randomized, open-label studies that had BMD and bone turnover variations as primary endpoints, ${ }^{12,13}$ was performed. The change in treatment satisfaction was assessed using the Treatment Satisfaction Questionnaire for Medication (TSQM), a tool validated for the measure of patient satisfaction with treatments of different chronic diseases and which consists of 14 items to assess an individual's perception of four domains of treatment satisfaction: 1) effectiveness, 2) side effects, 3) convenience, and 4) global satisfaction. ${ }^{30}$ The results of the study showed that osteoporotic postmenopausal women suboptimally adherent with oral daily or weekly bisphosphonate therapy, who switched to Dmab or monthly bisphosphonate treatment, reported greater satisfaction in all four domains of TSQM in both treatment groups at 6 and 12 months, but that these positive changes were significantly greater in patients in the Dmab group compared to those in patients in the monthly bisphosphonate group at all post-baseline time points. ${ }^{29}$

Whereas patient preference to 6-monthly Dmab injections versus oral weekly or monthly bisphosphonates was not surprising in relation to the more acceptable route of administration and the less frequent dosing regimen of the 6-monthly treatment option, patient preference between Dmab and another long-acting injectable therapy, such as zoledronic acid, could be less obvious. However, while several studies clearly demonstrated that patients preferred once yearly intravenous infusion of zoledronic acid rather than oral weekly bisphosphonates, ${ }^{31-33}$ a direct comparison in terms of patient satisfaction between Dmab and zoledronic acid for the treatment of postmenopausal osteoporosis is lacking. A recent retrospective study on a limited cohort of patients reported a statistically similar patient satisfaction between a group of patients treated with Dmab and another one treated with zoledronic acid,${ }^{34}$ but the small sample size and the design of the study (ie, each patient experienced only one of the two treatments without any experience of the other treatment) do not permit us to draw clear conclusions.

Other parameters closely related to treatment satisfaction and preference, which could influence patient medicationtaking behavior, are patient perceptions about a therapy in terms of the perceived necessity of the prescribed medication to treat a specific condition and concerns about potential adverse effects. A validated tool to assess these beliefs and concerns can be found in the Beliefs about Medicines Questionnaire (BMQ), which consists of 22 questions in the 
following major domains: 1) the necessity of the prescribed medication to treat osteoporosis in that moment or in the future; 2) concerns about potential side effects of taking the prescribed medication; and 3) preference for one drug over the other. ${ }^{35}$

At baseline in the DAPS study, ${ }^{27,28}$ when women were naïve to therapy, necessity and concerns scores were similar between groups. Subsequently, subject beliefs about the necessity for the prescribed treatment were significantly higher for Dmab than for alendronate at 6 months, but not at the following visits. Subject concerns about potential side effects were significantly lower for Dmab than for alendronate at the follow-up visits after the cross-over, when patients had experienced both forms of treatment administration, but not at previous time points. ${ }^{27,28}$ These variations in subject perceptions about treatment resulted in a significantly higher necessity-concerns differential (NCD) (ie, how much treatment necessity outweighs treatment concerns) for Dmab compared with alendronate at 6 months for both treatment years. ${ }^{36}$ Finally, the BMQ survey in the DAPS study provided significantly lower mean preference scores for alendronate than for Dmab at every visit, consistent with the preference scores of the PSQ. ${ }^{27,28}$

\section{Adherence to Dmab}

Many of the studies, which investigated preference for and satisfaction with Dmab, also evaluated adherence to the treatment, overall or in comparison with oral bisphosphonates, especially alendronate. Unfortunately, studies specifically designed to compare adherence to Dmab versus zoledronic acid are still lacking.

In the DECIDE and the STAND studies, where participants were strictly followed up every 3 months, compliance at 12 months (both injections received and $\geq 80 \%$ of the oral tablets) was 93\% and 94\%, respectively, with Dmab and 91\% and $94 \%$, respectively, with alendronate..$^{9,10}$

In the DAPS study, adherence, ie, both compliance (both Dmab injections 6 months \pm 4 weeks apart or $\geq 80 \%$ of alendronate tablets taken) and persistence (both Dmab injections or greater than two alendronate doses in the last month and completion of the treatment period), was assessed separately for each treatment year. ${ }^{27,28}$ By the end of the first 12 months, $88.1 \%$ of postmenopausal women were adherent to Dmab and $76.6 \%$ of patients were adherent to alendronate, while after the crossover, the adherence rate was $92.5 \%$ for Dmab and $63.5 \%$ for alendronate. A $46 \%$ and $80 \%$ relative risk reduction of nonadherence was calculated with Dmab compared to alendronate in the first and in the second year, respectively. ${ }^{27,28}$ The increase of nonadherence for alendronate-treated subjects after the crossover from Dmab, and conversely, the further decrease of nonadherence for Dmabtreated subjects after the crossover from alendronate, suggest a possible treatment sequence effect: a weekly dosing frequency may be more difficult to follow after a biannual dosing frequency than the opposite treatment sequence. DAPS investigators also examined whether the subjects' perception of their osteoporosis treatment and the treatment preferences influenced adherence. ${ }^{36}$ They found that at the beginning of the second year of treatment participant perception, as measured by BMQ scores, was a significant predictor of nonadherence. Indeed, as necessity scores increased, the odds of nonadherence decreased, and conversely, as concerns scores increased, the odds of nonadherence increased. These trends were reflected in the NCD. Indeed, higher NCD scores were significantly associated with lower odds of nonadherence, thus suggesting that positive perceptions of treatment positively influence medication-taking behavior. ${ }^{36}$

By extension, it seems feasible that understanding the factors that influence patient perceptions of osteoporosis treatments may result in an improved educational effort to increase adherence. In relation to this hypothesis, a successive study, although with several limitations, showed that in osteoporotic patients starting with a first Dmab injection, a positive feedback given to the patient already 6 months thereafter, based on the demonstration through a careful medical explanation of a rapid and highly significant BMD increase and on a good safety profile, was able to guarantee in $99 \%$ of patients the willingness to accept a second injection, thus reinforcing the role of patient perceptions and of their assessment during doctor-patient interactions on adherence to treatment. ${ }^{37}$

Several studies on medication-taking behavior of patients receiving Dmab are now ongoing, and data available so far confirm a high adherence to this 6-monthly subcutaneous treatment. ${ }^{38,39}$ The 12-month interim results of a European noninterventional study involving Germany, Austria, Greece, and Belgium showed that $82.7 \%-89.3 \%$ of patients received a second Dmab injection within 6 months \pm 4 weeks, and therefore, these patients were considered adherent to treatment, ${ }^{38} \mathrm{a}$ proportion of patients significantly greater than that observed in similar studies on bisphosphonates. ${ }^{17,19}$ In all four countries, these percentages increased as the permissible time window was extended, and up to $95.3 \%$ of patients received a second administration within 6 months \pm 8 weeks. ${ }^{38}$ At baseline, all participants completed the Morisky 8-Item Medication Adherence Scale (MMAS-8) questionnaire, a tool used to measure the probability of adherence. ${ }^{40}$ However, although 
the majority of patients had a low or medium score for adherence to prior treatments, their adherence to Dmab was high anyway, suggesting that some features of Dmab, such as the dosing schedule, may have positively influenced adherence behavior. No baseline variables were found to be significantly associated with persistence in the four countries, probably because of the high percentages observed and because of the different health care systems in the individual countries; nevertheless, in some countries, several significant associations were identified. Indeed, parental history of hip fracture was associated with higher persistence, while increased age, decreased mobility, and increased distance to the clinic were correlated with lower persistence. ${ }^{38}$

Finally, interim results of a 24-month multicenter, prospective, single-arm observational study in the US and Canada showed that at 12 months, $81.9 \%$ of patients were persistent with Dmab (ie, they received a second Dmab injection within 6 months \pm 8 weeks). ${ }^{39}$ As already described in another study, ${ }^{38}$ this percentage changed as the window was modified (from $74.8 \%$ of patients with a 4 -week window to $84.8 \%$ of patients with a 12 -week window). In this population, several baseline variables were found to be significantly associated with persistence among US patients and others among Canadian patients. ${ }^{39}$ In particular, it is worth signaling that US patients with greater NCD obtained by means of BMQ had a higher odds ratio for persistence, ${ }^{32}$ further confirming the role of patient perceptions of a specific medication in influencing medication-taking behavior, as already described in previous studies. . $3,36,37^{-3}$

\section{Limitations}

Several limitations must be considered in the interpretation of these trials. Firstly, participants enrolled in a clinical trial may differ from patients seen in real-life clinical practices. This difference may be observable to a greater extent in randomized clinical trials, where patients are regularly followed up by a skilled health care professional according to a precise study protocol. However, the participation in any prospective study, even observational, may influence patients' behavior, potentially leading to an overestimation of adherence to and preference for a specific treatment. This observation is supported by the finding that the rates of bisphosphonate adherence observed in the studies cited so far are meaningfully higher than rates observed in previous retrospective observational reports (less than half in the first year). ${ }^{17,19}$ The main reasons that may explain this phenomenon are the willingness of subjects to participate in a study and thus to accept the treatment offered, and the awareness of patients that their medication-taking behavior is being monitored. These two factors, differently from real-world behaviors, eliminate primary nonadherence patient behaviors in trials (ie, the refusal of a medication at first prescription). Moreover, in the clinical trials, participants are selected according to given inclusion criteria, and this aspect may limit the generalizability of results.

Secondly, in many of the cited studies, ${ }^{26-29,36-39}$ a conflict of interest cannot be excluded, and the provision of a drug to study participants by the study sponsor may have concealed the possible influences of treatment cost and accessibility on patient preference and adherence. Nevertheless, although several studies suggested that Dmab is cost-effective as compared to bisphosphonates, ${ }^{41-45}$ it must be considered that the effect of these aspects may be very difficult to assess since health care and reimbursement systems vary extensively between countries, and thus studies performed in different regions of the world become scarcely comparable.

Thirdly, the use of 1- or 2-year treatment periods in the cited trials ${ }^{27,28,38,39}$ may limit conclusions about long-term adherence to treatment, although a previous meta-analysis on bisphosphonate treatment suggests that nonadherence usually occurs shortly after treatment initiation. ${ }^{19}$

\section{Conclusion}

In spite of these limitations, available data suggest that, in comparison with bisphosphonates, postmenopausal women report greater preference to and satisfaction with Dmab, both overall and with its dosing frequency and route of administration, and they would choose Dmab over bisphosphonates for long-term osteoporosis treatment. ${ }^{26-29}$ Moreover, patient beliefs about the necessity of osteoporosis treatment and patient concerns about potential adverse events appear higher and lower, respectively, with Dmab than with bisphosphonates. This situation results in greater NCD scores, indicating that in patient perceptions, treatment necessity clearly outweighs concerns about Dmab. ${ }^{27,28,36}$ Furthermore, clinical trials showed that adherence among women treated with Dmab was consistently $>80 \%$ across studies; $9,10,13,27,28,37-39$ therefore, significantly higher adherence was shown in women treated with Dmab than in women treated with bisphosphonates, even when bisphosphonates were administered once monthly or intravenously. ${ }^{17,19-22}$ This different medication-taking behavior appears to be even more pronounced in patients previously treated, often sub-optimally, with bisphosphonates. ${ }^{12,13,28,38}$

Although in clinical trials an overestimation of adherence is conceivable, it must be considered that the adherence to 
Dmab requires only a 6-monthly visit, while the adherence to oral bisphosphonates requires the patient self-administration of tablets according to a correct dosing schedule and in the correct way. Therefore, it is possible that in clinical practice, the differences between Dmab and oral bisphosphonates in term of compliance and persistence may be even higher, although additional real-life studies are needed to confirm this assumption. Moreover, patient perception about treatment seems to influence medication-taking behavior, ${ }^{36,39}$ and during treatment follow-up, positive reinforcement based on the evidence of actual successful treatment outcomes, such as the BMD increase ${ }^{9-13}$ or bone turnover suppression, ${ }^{9-12}$ could help to further improve patient adherence. ${ }^{37}$ Indeed, due to the asymptomatic nature of osteoporosis, until a fracture occurs or even later, the patient could easily underestimate the importance of osteoporosis medication, resulting in poor adherence and therefore an increased risk of fracture.

In conclusion, current evidence underlines the necessity to personalize osteoporosis treatment, taking patient preference into account, especially in regards to frequency and route of administration. Current evidence also draws attention to patient beliefs at the initiation of therapy and during follow-up. These efforts are addressed to improve adherence to osteoporosis treatment and, as a consequence, to achieve more successful treatment outcomes, thus positively impacting on the cost-effectiveness of the chosen drug. ${ }^{45}$ From this perspective, according to the data demonstrating a better adherence to Dmab compared to other osteoporosis treatments, especially bisphosphonates, Dmab may represent a reasonable and effective alternative to bisphosphonates, particularly for osteoporotic women in whom a suboptimal or even poor adherence to oral treatments is expected.

Further studies are required in the future to assess long-term adherence and preference to Dmab in real-world clinical practices, to evaluate its long-term safety, and to assess its effectiveness as compared head-to-head with bisphosphonates.

\section{Disclosure}

The authors report no conflicts of interest in this work.

\section{References}

1. Boyle WJ, Simonet WS, Lacey DL. Osteoclast differentiation and activation. Nature. 2003;423(6937):337-342.

2. Bone HG, Bolognese MA, Yuen CK, et al. Effects of denosumab on bone mineral density and bone turnover in postmenopausal women. J Clin Endocrinol Metab. 2008;93:2149-2157.

3. Lewiecki EM, Miller PD, McClung MR, et al. Two-year treatment with denosumab (AMG 162) in a randomized phase 2 study of postmenopausal women with low BMD. J Bone Miner Res. 2007;22:1832-1841.
4. McClung MR, Lewiecki EM, Cohen SB, et al; AMG 162 Bone Loss Study Group. Denosumab in postmenopausal women with low bone mineral density. $N$ Engl J Med. 2006;354:821-831.

5. Miller PD, Bolognese MA, Lewiecki EM, et al; Amg Bone Loss Study Group. Effect of denosumab on bone density and turnover in postmenopausal women with low bone mass after long-term continued, discontinued, and restarting of therapy: a randomized blinded phase 2 clinical trial. Bone. 2008;43:222-229.

6. Cummings SR, San Martin J, McClung MR, et al; FREEDOM Trial. Denosumab for prevention of fractures in postmenopausal women with osteoporosis. N Engl J Med. 2009;361(8):756-765.

7. Papapoulos S, Lippuner K, Roux C, et al. The effect of 8 or 5 years of denosumab treatment in postmenopausal women with osteoporosis: results from the FREEDOM Extension study. Osteoporos Int. Epub $2015 \mathrm{Jul} 23$.

8. Rachner TD, Khosla S, Hofbauer LC. Osteoporosis: now and the future. Lancet. 2011;377(9773):1276-1287.

9. Kendler DL, Roux C, Benhamou CL, et al. Effects of denosumab on bone mineral density and bone turnover in postmenopausal women transitioning from alendronate therapy. J Bone Miner Res. 2010;25(1): $72-81$.

10. Brown JP, Prince RL, Deal C, et al. Comparison of the effect of denosumab and alendronate on BMD and biochemical markers of bone turnover in postmenopausal women with low bone mass: a randomized, blinded, phase 3 trial. J Bone Miner Res. 2009;24(1):153-161.

11. Brown JP, Roux C, Ho PR, et al. Denosumab significantly increases bone mineral density and reduces bone turnover compared with monthly oral ibandronate and risedronate in postmenopausal women who remained at higher risk for fracture despite previous suboptimal treatment with an oral bisphosphonate. Osteoporos Int. 2014;25(7):1953-1961.

12. Roux C, Hofbauer LC, Ho PR, et al. Denosumab compared with risedronate in postmenopausal women suboptimally adherent to alendronate therapy: efficacy and safety results from a randomized open-label study. Bone. 2014;58:48-54.

13. Recknor C, Czerwinski E, Bone HG, et al. Denosumab compared with ibandronate in postmenopausal women previously treated with bisphosphonate therapy: a randomized open-label trial. Obstet Gynecol. 2013;121(6):1291-1299.

14. Murad MH, Drake MT, Mullan RJ, et al. Clinical review. Comparative effectiveness of drug treatments to prevent fragility fractures: a systematic review and network meta-analysis. J Clin Endocrinol Metab. 2012; 97(6):1871-1880.

15. Cummings SR, Melton LJ. Epidemiology and outcomes of osteoporotic fractures. Lancet. 2002;359(9319):1761-1767.

16. Cramer JA, Roy A, Burrell A, et al. Medication compliance and persistence: terminology and definitions. Value Health. 2008;11(1):44-47.

17. Imaz I, Zegarra P, González-Enríquez J, Rubio B, Alcazar R, Amate JM. Poor bisphosphonate adherence for treatment of osteoporosis increases fracture risk: systematic review and meta-analysis. Osteoporos Int. 2010; 21(11):1943-1951.

18. Hiligsmann M, Rabenda V, Gathon HJ, Ethgen O, Reginster JY. Potential clinical and economic impact of nonadherence with osteoporosis medications. Calcif Tissue Int. 2010;86(3):202-210.

19. Kothawala P, Badamgarav E, Ryu S, Miller RM, Halbert RJ. Systematic review and meta-analysis of real-world adherence to drug therapy for osteoporosis. Mayo Clin Proc. 2007;82(12):1493-1501.

20. Lee YK, Nho JH, Ha YC, Koo KH. Persistence with intravenous zoledronate in elderly patients with osteoporosis. Osteoporos Int. 2012; 23(9):2329-2333.

21. Curtis JR, Yun H, Matthews R, Saag KG, Delzell E. Adherence with intravenous zoledronate and intravenous ibandronate in the United States Medicare population. Arthritis Care Res (Hoboken). 2012;64(7): 1054-1060.

22. Ziller V, Kostev K, Kyvernitakis I, Boeckhoff J, Hadji P. Persistence and compliance of medications used in the treatment of osteoporosis analysis using a large scale, representative, longitudinal German database. Int J Clin Pharmacol Ther. 2012;50(5):315-322. 
23. Barrett-Connor E, Wade SW, Do TP, et al. Treatment satisfaction and persistence among postmenopausal women on osteoporosis medications: 12-month results from POSSIBLE US ${ }^{\mathrm{TM}}$. Osteoporos Int. 2012;23(2): $733-741$.

24. Reginster JY, Rabenda V, Neuprez A. Adherence, patient preference and dosing frequency: understanding the relationship. Bone. 2006;38 (4 Suppl 1):S2-S6.

25. Gold DT, Horne R, Coon CD, et al. Development, reliability, and validity of a new Preference and Satisfaction Questionnaire. Value Health 2011;14(8):1109-1116.

26. Kendler DL, Bessette L, Hill CD, et al. Preference and satisfaction with a 6-month subcutaneous injection versus a weekly tablet for treatment of low bone mass. Osteoporos Int. 2010;21(5):837-846.

27. Kendler DL, McClung MR, Freemantle N, et al; DAPS Investigators. Adherence, preference, and satisfaction of postmenopausal women taking denosumab or alendronate. Osteoporos Int. 2011;22(6):1725-1735.

28. Freemantle N, Satram-Hoang S, Tang ET, et al; DAPS Investigators. Final results of the DAPS (Denosumab Adherence Preference Satisfaction) study: a 24-month, randomized, crossover comparison with alendronate in postmenopausal women. Osteoporos Int. 2012;23(1): 317-326.

29. Palacios S, Agodoa I, Bonnick S, et al. Treatment satisfaction in postmenopausal women suboptimally adherent to bisphosphonates who transitioned to denosumab compared with risedronate or ibandronate. $J$ Clin Endocrinol Metab. 2015;100(3):E487-E492.

30. Atkinson MJ, Sinha A, Hass SL, et al. Validation of a general measure of treatment satisfaction, the Treatment Satisfaction Questionnaire for Medication (TSQM), using a national panel study of chronic disease. Health Qual Life Outcomes. 2004;2:12.

31. McClung M, Recker R, Miller P, et al. Intravenous zoledronic acid $5 \mathrm{mg}$ in the treatment of postmenopausal women with low bone density previously treated with alendronate. Bone. 2007;41(1):122-128.

32. Saag K, Lindsay R, Kriegman A, Beamer E, Zhou W. A single zoledronic acid infusion reduces bone resorption markers more rapidly than weekly oral alendronate in postmenopausal women with low bone mineral density. Bone. 2007;40(5):1238-1243.

33. Lee S, Glendenning P, Inderjeeth CA. Efficacy, side effects and route of administration are more important than frequency of dosing of antiosteoporosis treatments in determining patient adherence: a critical review of published articles from 1970 to 2009. Osteoporos Int. 2011; 22(3):741-753.

34. Sheedy KC, Camara MI, Camacho PM. Comparison of the efficacy, adverse effects, and cost of zoledronic acid and denosumab in the treatment of osteoporosis. Endocr Pract. 2015;21(3):275-279.
35. Horne R, Weinman J, Hankins M. The Beliefs about Medicines Questionnaire (BMQ): a new method for assessing cognitive representations of medication. Psychol Health. 1999;10:1-29.

36. Kendler DL, Macarios D, Lillestol MJ, et al. Influence of patient perceptions and preferences for osteoporosis medication on adherence behavior in the Denosumab Adherence Preference Satisfaction study. Menopause. 2014;21(1):25-32.

37. Ringe JD, Farahmand P. Improved real-life adherence of 6-monthly denosumab injections due to positive feedback based on rapid 6-month BMD increase and good safety profile. Rheumatol Int. 2014;34(5): 727-732.

38. Hadji P, Papaioannou N, Gielen E, et al. Persistence, adherence, and medication-taking behavior in women with postmenopausal osteoporosis receiving denosumab in routine practice in Germany, Austria, Greece, and Belgium: 12-month results from a European noninterventional study. Osteoporos Int. Epub 2015 May 28.

39. Silverman SL, Siris E, Kendler DL, et al. Persistence at 12 months with denosumab in postmenopausal women with osteoporosis: interim results from a prospective observational study. Osteoporos Int. 2015;26(1): $361-372$.

40. Morisky DE, Ang A, Krousel-Wood M, Ward HJ. Predictive validity of a medication adherence measure in an outpatient setting. $J$ Clin Hypertens (Greenwich). 2008;10(5):348-354.

41. Hiligsmann M, Reginster JY. Cost effectiveness of denosumab compared with oral bisphosphonates in the treatment of post-menopausal osteoporotic women in Belgium. Pharmacoeconomics. 2011;29(10): 895-911.

42. Parthan A, Kruse M, Yurgin N, Huang J, Viswanathan HN, Taylor D. Cost effectiveness of denosumab versus oral bisphosphonates for postmenopausal osteoporosis in the US. Appl Health Econ Health Policy. 2013; 11(5):485-497.

43. Chau D, Becker DL, Coombes ME, Ioannidis G, Adachi JD, Goeree R. Cost-effectiveness of denosumab in the treatment of postmenopausal osteoporosis in Canada. J Med Econ. 2012;15(Suppl 1):3-14.

44. Hiligsmann M, Boonen A, Dirksen CD, Ben Sedrine W, Reginster JY. Cost-effectiveness of denosumab in the treatment of postmenopausal osteoporotic women. Expert Rev Pharmacoecon Outcomes Res. 2013; 13(1):19-28

45. Jönsson B, Ström O, Eisman JA, et al. Cost-effectiveness of Denosumab for the treatment of postmenopausal osteoporosis. Osteoporos Int. 2011; 22(3):967-982.
International Journal of Women's Health

\section{Publish your work in this journal}

The International Journal of Women's Health is an international, peerreviewed open-access journal publishing original research, reports, editorials, reviews and commentaries on all aspects of women's healthcare including gynecology, obstetrics, and breast cancer. The manuscript management system is completely online and includes

\section{Dovepress}

a very quick and fair peer-review system, which is all easy to use. Visit http://www.dovepress.com/testimonials.php to read real quotes from published authors. 